Pacific

Journal of

Mathematics

\title{
BORDISM AND REGULAR HOMOTOPY OF LOW-DIMENSIONAL IMMERSIONS
}

JOHN FORBES HugHES

Volume $156 \quad$ No. 1

November 1992 


\title{
BORDISM AND REGULAR HOMOTOPY OF LOW-DIMENSIONAL IMMERSIONS
}

\author{
JOHN Forbes Hughes
}

In this paper we study the geometric characteristics of low-dimensional immersions. Smale asked, in his paper on immersions of the $k$-sphere in $R^{n}$, what are explicit generators for the groups of regular homotopy classes of immersions? We answer this for the 3-sphere in $R^{4}$ and $R^{5}$. For $S^{3}$ in $R^{4}$, the answer is:

THEOREM. The standard (Froissart-Morin) eversion of $S^{2}$ in $R^{3}$ has, as a track, an immersion of $S^{2} \times I$ in $R^{4}$ whose ends are embedded $S^{2}$ s. Each of these bounds a 3-ball in $R^{4}$. Capping off the track with these 3-balls yields an immersion $K: S^{3} \rightarrow R^{4}$. Performing the eversion twice and capping off gives an immersion $E: S^{3} \rightarrow R^{4}$. The immersions $E$ and $K$ generate the group of regular homotopy classes of immersions of $S^{3}$ in $R^{4}$.

We also relate the invariants of an immersion which bounds an immersion of a manifold of one higher dimension to the characteristic classes of that manifold.

1. Notation, definitions, and preliminary results. We begin by establishing our notation and definitions. All manifolds and maps in this paper are smooth, unless otherwise noted.

DEFINITION. A $k$-frame in a vector space is an ordered $k$-tuple of linearly independent vectors $v^{1}, \ldots, v^{k}$. A $k$-frame in a $k$-dimensional space may be considered an ordered basis of the space. Frames are denoted by square brackets, and we implicitly extend any maximal rank linear map between vector spaces $V$ and $W$ to also take frames in $V$ to frames in $W$, by defining

$$
T\left[v^{1}, \ldots, v^{k}\right]=\left[T v^{1}, \ldots, T v^{k}\right] .
$$

Definition. $V_{n, k}$ denotes the Stiefel manifold of $k$-frames in $R^{n}$. If $N$ is an $n$-plane bundle, $V_{k}(N)$ denotes the associated bundle of $k$-frames.

Notation. $T M$ denotes the tangent bundle of the manifold $M$. The bundle projection is denoted by $\pi$. For a typical point $p$ of $M$, the fiber over $p, \pi^{-1}(p)$, is denoted by $T_{p} M$. 
A tangent vector at $p$ is an element of $T_{p} M$, and is typically written with a subscript to indicate this: $v_{p}$.

If $p$ is a point of a parallelizable $n$-manifold and a parallelization has been fixed, tangent vectors at $p$ may be identified with the corresponding vectors in $R^{n}$ and written $(v ; p)$, where $w$ is a vector in $R^{n}$.

If $p$ is a point of a manifold $M^{n}$, then a frame at $p$ is a frame in the tangent space to $M$ at $p$, i.e., an $n$-tuple of linearly independent vectors $\left[v_{p}^{1}, \ldots, v_{p}^{n}\right]$. In the event that the manifold is parallelizable and a parallelization $\phi: M \times R^{n} \rightarrow T M:(p, v) \rightarrow \phi(p, v)_{p}$ has been fixed, the frame $\left[\phi\left(p, v^{1}\right)_{p}, \ldots, \phi\left(p, v^{n}\right)_{p}\right]$, at $p$ may be written

$$
\left[v^{1}, \ldots, v^{n} ; p\right] \text {, }
$$

where $v^{1}, \ldots, v^{n}$ are vectors in $R^{n}$.

Matrices are written in terms of their columns, for example

$$
A=\left[A^{1}, \ldots, A^{n}\right]
$$

indicates that $A$ is a matrix with columns $A^{1}$ through $A^{n}$. We will often consider a matrix as a frame made of its columns, or a frame as a matrix whose columns are the vectors of the frame.

Convention. In homotopy calculations, basepoints are often required. The basepoint for every matrix group is the identity matrix, written $I_{n}$ (for the $n$ by $n$ identity). The basepoint for $V_{n, k}$ is the frame $\left[e^{1}, \ldots, e^{k}\right]$.

Definition. If $M^{n}$ is an oriented manifold with boundary, then an outward normal is any vector $v$ such that there is a curve $\gamma$ in $M$ with

(a) $\gamma(x) \in M-\partial M$ for $x \in[0,1)$,

(b) $\gamma(1) \in \partial M$

(c) $v=\gamma^{\prime}(1) \notin T_{\gamma(1)}(\partial M)$.

If $p \in \partial M$, then $T_{n}(\partial M)$ is a codimension 1 subspace of $T_{p} M$. Let $\left[v_{p}^{2}, \ldots, v_{p}^{n}\right]$ be an ordered basis of $T_{p}(\partial M)$ and $v_{p}^{1}$ be an outward normal vector at $p$. Then $\left[v_{p}^{2}, \ldots, v_{p}^{n}\right]$ is a positively oriented basis of $T_{p}(\partial M)$ if and only if $\left[v_{p}^{1}, \ldots, v_{p}^{n}\right]$ is a positively oriented basis of $T_{p} M$. This defines the orientation of $\partial M$ induced by the orientation of $M$.

DEFINITION. If $\xi$ and $\mu$ are vector bundles over manifolds $M$ and $N$ respectively, then $\operatorname{Hom}(\xi, \mu)$ is the fiber bundle over $M$ whose 
fiber at $p$ consists of all linear maps from $\xi_{p}$, the fiber of $\xi$ at $p$, to $\mu$, where by a linear map we mean a linear map from $\xi_{p}$ to some fiber of $\mu$.

If $f: M \rightarrow N$ is a smooth map between manifolds, we denote its derivative by $T f$. The map $T f$ is a section of $\operatorname{Hom}(T M, T N)$, so that for each point $p, T f(p)$ is a linear map from $T_{p} M$ to $T_{f(p)} N$. Henceforth, we will write $T_{p} f$ instead of $T f(p)$.

Definition. If $M$ and $K$ are manifolds of dimensions $m$ and $k$, respectively, then a map $f: M \rightarrow K$ is an immersion if $T_{p} f: T_{p} M \rightarrow$ $T_{f(p)} K$ has rank $m$ (i.e., is injective) for every point $p$ in $M$.

Definition. An immersion $f: M^{n} \rightarrow K^{k}$ is a generic immersion if whenever $y \in K$ and $f^{-1}(y)=\left\{p_{1}, \ldots, p_{r}\right\}$, there are the following data $(k \geq n)$ :

(i) a $(k)$-ball neighborhood $B(y)$ of $y$,

(ii) a diffeomorphism $\omega: B(y) \rightarrow R^{k}$, and

(iii) an $n$-disk neighborhood $D\left(p_{j}\right)$ of $p_{j}$ such that

$$
\left.\omega \circ f\right|_{D\left(p_{j}\right)}: D\left(p_{j}\right) \rightarrow R^{k}
$$

has as its image the hyperplane of $R^{k}$ which is orthogonal to $e^{1+(j-1)(k-n)}, \ldots, e^{j(k-n)}$. If $k=n$, an immersion is called generic if either $\partial M=\phi$ or $f \mid \partial M$ is generic in the sense above.

REMARK. Since the differential has maximal rank, it induces a map on frames, i.e., if $f: M^{m} \rightarrow K^{k}$ is an immersion, then $T f$ induces a map from $V_{m}(T M)$ to $V_{m}(T K)$ (see [Fra71]).

Definition. If $H: X \times[0,1] \rightarrow Y$ is a homotopy, the track of $H$ is the map

$$
\bar{H}: X \times[0,1] \rightarrow Y \times[0,1]:(x, t) \rightarrow(H(x, t), t) .
$$

The word track is sometimes used to denote the image of $\bar{H}$ as well.

DEFINITION. If $f$ and $g$ are immersions of $M^{m}$ into $K^{k}$, then $f$ and $g$ are regularly homotopic if there is a homotopy

$$
H: M \times I \rightarrow K
$$

such that the track of $H$ is an immersion of $M \times I$ in $K \times I . H$ is called a regular homotopy of $f$ to $g$. 
Proposition 1.1. If $f$ and $g$ are regularly homotopic immersions of $M^{m}$ into $K^{k}$, then the tangent maps, $T f$ and $T g$, are homotopic sections of $\operatorname{Mono}(T M, T K)$, the subbundle of $\operatorname{Hom}(T M, T K)$ consisting of maps injective on each fiber.

Proof. Let $H$ be the regular homotopy from $f$ to $g$. Then the derivative of $H$ is the homotopy from $T f$ to $T g$.

Definition. We will often need to refer to various points and regions of the $k$-sphere. The point $(0, \ldots, 1)$ is called the north pole; the point $(0, \ldots,-1)$ is the south pole. The points $\left(x_{0}, \ldots, x_{k}\right)$ with $x_{k}>0$ constitute the northern hemisphere, which is denoted $S_{+}^{k}$; those with $x_{k}<0$ are the southern hemisphere, denoted $S_{-}^{k}$. The arctic and antarctic regions are those parts of the sphere where $x_{k}>1 / 2$ and $x_{k}<1 / 2$, respectively, and the temperate zone is the region $-1 / 2<x_{k}<1 / 2$.

Definition. An immersion $f: S^{k} \rightarrow R^{n}$ is said to be a good immersion if the $f$ agrees with the standard embedding of $S^{k}$ in $R^{n}$ on some neighborhood of the south pole. (The standard embedding of $S^{k}$ in $R^{n}$ is given by the inclusion of $R^{k+1}$ into $R^{n}$ by appending $n-(k+1)$ zeroes to each point.)

REMARK. Any good immersion $f$ that agrees with the standard one on a neighborhood of the south pole (say on $\left\{\left(x_{0}, \ldots, x_{k}\right) \mid x_{k}<-a\right\}$, where $0<a<1)$ is regularly homotopic to one that agrees with the standard embedding on the entire southern hemisphere, which can be seen by considering $g_{t}(x)=H_{t}\left(f\left(H_{t}^{-1}(x)\right)\right)$, where $H_{t}: S^{k} \rightarrow S^{k}:\left(x_{0}, \ldots, x_{k}\right) \rightarrow\left(x_{0}, \ldots, x_{k}+a t\right) /\left\|\left(x_{0}, \ldots, x_{k}+a t\right)\right\|$.

Clearly $H_{0}$ is the identity, and hence $g_{0}=f$. But $g_{1}$ agrees with the standard embedding on the entire southern hemisphere, and is homotopic to $f$ though the maps $g_{t}, t \in[0,1]$.

The assumption that an immersion is good guarantees that its differential takes the standard frame for the tangent plane to the south pole to the standard $k$-frame in the tangent space to $R^{n}$ at the image of the south pole. This is needed for basepoints in homotopy. computations. It will turn out to be unimportant, since most of the homotopy groups are of topological groups, hence abelian and basepoint independent. All codimension one immersions of spheres will be assumed good unless otherwise noted. Even higher codimension 
immersions may be assumed good without loss of generality (see the remark following Theorem 1.4).

Definition. If $f: S^{k} \rightarrow R^{n}$ is a good immersion, and [ $\left.f\right]$ denotes its regular homotopy class, then the immersion invariant $I[f]$, is defined as follows:

First adjust $f$ to be good on $S_{-}^{k}$. Since the northern hemisphere of $S^{k}, N$, is a disk, we fix a section, $\sigma$, of the trivial bundle $V_{k}(T N)$, i.e., a framing of the tangent bundle over the northern hemisphere. Pick $\sigma$ so that $\sigma$ (northpole) is the standard $k$-frame for the tangent space to $R^{k}$ at the north pole. Let us denote by

$$
i: S^{k} \rightarrow R^{k+1} \rightarrow R^{n}:\left(x_{0}, \ldots, x_{k}\right) \rightarrow\left(x_{0}, \ldots, x_{k}, 0, \ldots, 0\right)
$$

the standard inclusion.

Now,

$$
T f \circ \sigma: N \rightarrow V_{k}(T N) \rightarrow V_{k}\left(T R^{n}\right)
$$

and

$$
T i \circ \sigma: N \rightarrow V_{k}(T N) \rightarrow V_{k}\left(T R^{n}\right)
$$

agree on the equator, $\partial N$. Let

$$
j: S^{k} \rightarrow S^{k}:\left(x_{0}, \ldots, x_{k}\right) \rightarrow\left(x_{0}, \ldots,-x_{k}\right)
$$

and let

$$
e: V_{k}\left(T R^{n}\right) \rightarrow V_{n, k}:\left[V^{1}, \ldots, V^{k} ; p\right] \rightarrow\left[V^{1}, \ldots, V^{k}\right] .
$$

Define

$$
I[f]= \begin{cases}e(T f \circ \sigma(p)), & p \in N, \\ e(T i \circ \sigma(j(p))), & p \in S^{k}-N .\end{cases}
$$

Then $I[f]$ is a map from $S^{k}$ to $V_{n, k} . I[f]$ maps the south pole to the frame $\left[e^{1}, \ldots, e^{k}\right]$; hence $I[f]$ represents an element of $\pi_{k}\left(V_{n, k}\right)$.

To justify the name "immersion invariant," we need a theorem due to Smale [Sma59]:

THEOREM 1.2 (Smale). $I[f]$ is well-defined on regular homotopy classes of good immersions, and the map I taking regular homotopy classes of (good) immersions of $S^{k}$ in $R^{n}$ to elements of $\pi_{k}\left(V_{n, k}\right)$ is an isomorphism of sets (i.e., it is 1-1 and onto) whenever $k<n$. In fact the space of maps from the pair $\left(S^{k}, S^{k}-N\right)$ to the pair $\left(V_{n, k},\left[e^{1}, \ldots, e^{k}\right]\right)$ is weak homotopy equivalent to the space of good 
immersions of $S^{k}$ in $R^{n}$, with the map I being the homotopy equivalence in one direction.

In the case of an $(n-1)$-sphere immersed in $R^{n}$, the situation may be slightly altered (and made a little simpler).

Definition (of $\bar{I}$ ). Given a good immersion $f: S^{n-1} \rightarrow R^{n}$, the normal bundle of $f$ is a line bundle over $S^{n-1}$, and is orientable, hence trivial. The tubular neighborhood theorem guarantees the existence of an extension of $f$ to a map $\bar{f}: S^{n-1} \times R^{n}$ which is also an immersion.

Furthermore, since $S^{n-1} \times I$ is standardly embedded as a subset of $R^{n}$, it inherits an orientation from $R^{n}$. One can choose $\bar{f}$ so that it is orientation preserving (if it is not, simply redefine it: $\bar{f}_{\text {new }}(x, t)=$ $\bar{f}(x,-t)$ for $\left.x \in S^{n}, t \in I\right)$.

The map $T_{p} \bar{f}$ pushes forward the frame $\left[e^{1}, \ldots, e^{n}\right]$ to a frame $\left[T_{p} \bar{f} e^{1}, \ldots, T_{p} \bar{f} e^{n}\right]$. Thus

$$
p \rightarrow\left[T_{p} \bar{f}\left(e_{p}^{1}\right), \ldots, T_{p} \bar{f}\left(e_{p}^{n}\right) ; f(p)\right]
$$

defines a map from $S^{n-1}$ to $V_{n}\left(T R^{n}\right)$, and sending $\left[v^{1}, \ldots, v^{n} ; p\right]$ to $\left[v^{1}, \ldots, v^{n}\right]$ yields a map

$$
I(f): S^{n-1} \rightarrow V_{n, n} .
$$

By applying the Gram-Schmidt process to elements of $V_{n, n}$ we may alter $I(f)$ to a map $\bar{I}(f): S^{n-1} \rightarrow \mathrm{SO}(n)$. Because $f$ is good, we know that $\bar{I}(f)$ (south pole) $=I_{n}$, the identity matrix. Thus $\bar{I}(f) \in$ $\pi_{n-1}(\mathrm{SO}(n))$.

REMARK. $\bar{I}(f)$ is, roughly, the map induced by the differential of the "tangent-plus-normal" map of $f$; evidently if $f$ and $g$ are regularly homotopic (rel the south pole), then $\bar{I}(f)=\bar{I}(g)$ in $\pi_{n-1}(\operatorname{SO}(n))$.

Definition. If $u^{1}, \ldots, u^{n-1}$ are vectors in $R^{n}$, then the cross product of $u^{1}, \ldots, u^{n-1}$ is the vector $w$ such that $w$ is orthogonal to $u^{1}, \ldots, u^{n-1}$ and

$$
\operatorname{det}\left[u^{1}, \ldots, u^{n-1}, w\right]=\|w\|^{2}
$$

and is written $u^{1} \times u^{2} \times \cdots \times u^{n-1}$.

REMARK. The map

$$
c: V_{n, n-1} \rightarrow V_{n, n}: u^{1}, \ldots, u^{n-1} \rightarrow u^{1}, \ldots, u^{n-1}, w,
$$



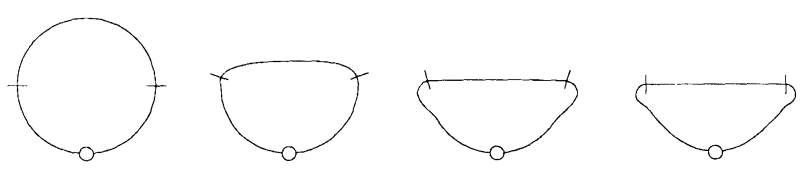

FIGURE 1.1

where $w=u^{1} \times \cdots \times u^{n-1}$, is a homotopy equivalence of $V_{n, n-1}$ with the component of $\left[e^{1}, \ldots, e^{n}\right]$ in $V_{n, m}$. Composing with the Gram-Schmidt process yields a homotopy equivalence from $V_{n, n}$ to $\mathrm{SO}(n)$, so that

$$
\pi_{n-1}\left(V_{n, n-1}\right) \simeq \pi_{n-1}(\mathrm{SO}(n))
$$

LEMMA 1.3. Under this isomorphism, $I[f]=\bar{I}(f)$.

Proof. Restating the lemma, we claim that $G \circ c(I[f])$ and $\bar{I}(f)$ are homotopic maps from $\left(S^{n-1}, \mathrm{SP}\right)$ to $(\mathrm{SO}(n), I)$, where $G$ denotes the Gram-Schmidt process and SP denotes the south pole. We prove this by altering each of these maps by a homotopy, and by making a good choice for $\sigma$ in the definition of $I[f]$. Assume that $f$ has been modified (as in the Remark following Proposition 1.1) to agree with the standard embedding on the southern hemisphere.

Step 1. Construct an isotopy $H_{t}: R^{n} \rightarrow R^{n}(t \in[0,1])$ such that

(1) A neighborhood of the south pole of $S^{n-1}$ remains fixed by $H_{t}$ for every $t$.

(2) Under $H_{1}$, a neighborhood of the northern hemisphere of $S^{n-1}$ is "flattened" onto a neighborhood of the unit disk of $R^{n-1} \subseteq$ $R^{n}$, in such a way that the northern hemisphere is sent to the unit disk.

Figure 1.1 shows this for $S^{1}$ in $R^{2}$. The hatch marks on the circle denote the division between the southern and northern hemisphere, i.e., the equator, and the dot denotes the south pole.

Step 2. Construct a map homotopic to $I[f]$ (see Figure 1.2 on next page). Let $\sigma$ be a framing of the tangent bundle of $S^{n-1}$ over the northern hemisphere, as in the definition of $I[f]$, and let

$$
I_{t}[f]= \begin{cases}e\left(T_{p}\left(H_{t} \circ f\right)(\sigma(p))\right), & p \in N, \\ e\left(T_{p}\left(H_{t} \circ i\right)(\sigma(j(p)))\right), & p \in S^{n-1}-N .\end{cases}
$$




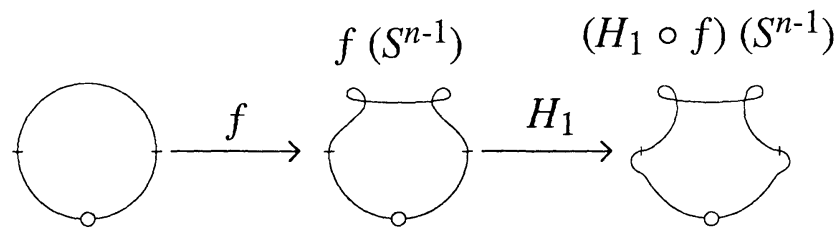

FIGURE 1.2

Clearly $I_{0}[f]=I[f]$, and $I_{1}[f]$ is homotopic to $I_{0}[f]$. Furthermore, since $H_{t}$ is the identity near the south pole (SP) of $S^{n-1}$, $I_{t}[f](\mathrm{SP})=\left[e_{H_{t} \circ f(p)}^{1}, \ldots, e_{H_{t} \circ f(p)}^{n-1}\right]$, and hence $G \circ c\left(I_{t}[f](\mathrm{SP})\right)=I$; thus $I_{t}$ provides a homotopy respecting basepoints.

Step 3. Choose a particular framing, $\sigma$, of the upper hemisphere. For each point $p \in N, H_{1}(p)$ lies in the $R^{n-1}$ plane of $R^{n}$, and the tangent space to $H_{1}\left(S^{n-1}\right)$ at $H_{1}(p)$ is just $R^{n-1}$; a natural frame there is $\left[e^{1}, \ldots, e^{n-1}\right]$. We pull this frame back to $S D^{n-1}$ by the differential of $H^{-1}$, i.e., we let

$$
\sigma(p)=\left(T_{H_{1}(p)} H_{1}^{-1}\right)\left[e_{H_{1}(p)}^{1}, \ldots, e_{H_{1}(p)}^{n-1}\right] .
$$

The expression for $I_{1}[f]$ is now

$$
I_{1}[f]=\left\{\begin{array}{l}
e\left(T_{p}\left(H_{1} \circ f\right)\left(T_{H_{1}(p)} H_{1}^{-1}\right)\left[e_{H_{1}(p)}^{1}, \ldots, e_{H_{1}(p)}^{n-1}\right]\right), \quad p \in N, \\
e\left(T_{p}\left(H_{1} \circ i\right)\left(\left(T_{H_{1}(j(p))} H_{1}^{-1}\right)\left[e_{H_{1}(j(p))}^{1}, \ldots, e_{H_{1}(j(p))}^{n-1}\right]\right)\right), \\
\quad p \in S^{n-1}-N .
\end{array}\right.
$$

Step 4. Construct a map isotopic to $\bar{I}(f)$. Recall that

$$
\bar{I}(f)(p)=G \circ e\left(T_{p} f\left[e_{p}^{1}, \ldots, e_{p}^{n}\right]\right) .
$$

If we let

$$
\bar{I}_{t}(f)(p)=G \circ e\left(T_{p}\left(H_{t} \circ f\right)\left[T_{H_{t}(p)} H_{t}^{-1}\left[e_{H_{t}(p)}^{1}, \ldots, e_{H_{t}(p)}^{n}\right]\right]\right)
$$

(see Figure 1.3) then

(a) $\bar{I}_{0}(f)=\bar{I}(f)$, because $H_{0}$ is the identity on $R^{4}$.

(b) $\bar{I}_{t}(f)(p)=\bar{I}(f)(p)=I$ for $p$ near the south pole, because near the south pole, $H_{t}$ is the identity for all $t$. 


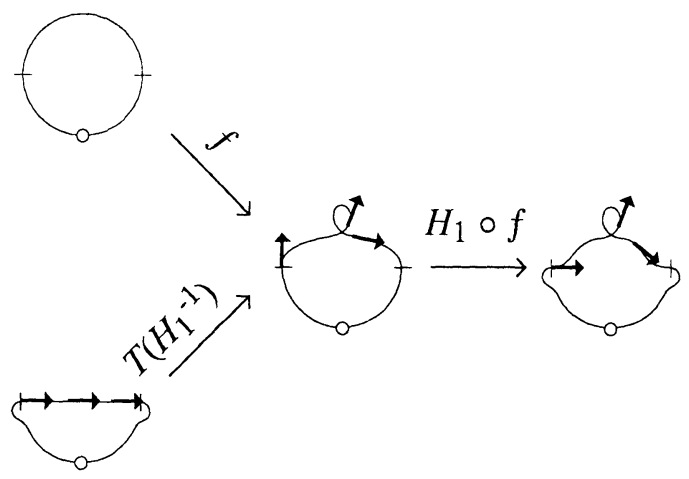

FIGURE 1.3

Hence $\bar{I}_{1}(f)$ and $\bar{I}(f)$ represent the same element of

$$
\pi_{n-1}(\mathrm{SO}(n), I) \text {. }
$$

In particular, the lemma now reduces to showing that $G \circ c\left(I_{1}[f]\right)$ and $\bar{I}_{1}(f)$ represent the same element of $\pi_{n-1}(\mathrm{SO}(n), I)$.

Step 5. Observe that $G \circ c\left(I_{1}[f]\right)$ and $\bar{I}_{1}(f)$ are identical, and thus prove the lemma.

Writing out the formula for $\bar{I}_{1}(f)$, we get

$$
\bar{I}_{1}(f)(p)=G \circ e\left(T_{p}\left(H_{1} \circ f\right)\left[T_{H_{1}(p)} H_{1}^{-1}\left[e_{H_{1}(p)}^{1}, \ldots, e_{H_{1}(p)}^{n}\right]\right]\right) .
$$

For $p \in N$, we have

$$
\begin{aligned}
& (G \circ c)\left(I_{1}[f](p)\right) \\
& \quad=(G \circ c \circ e)\left(T_{p}\left(H_{1} \circ f\right)\left[T_{H_{1}(p)} H_{1}^{-1}\left[e_{H_{1}(p)}^{1}, \ldots, e_{H_{1}(p)}^{n-1}\right]\right]\right) .
\end{aligned}
$$

These are evidently identical frames, at least for the first $n-1$ vectors. But the last vectors must agree as well, since both frames are positively oriented.

For $p \in S^{n-1}-N$, we have

$$
\begin{aligned}
(G \circ c) & \left(I_{1}[f](p)\right) \\
= & (G \circ c \circ e)\left(T_{p}\left(H_{1} \circ i\right)\left[T_{H_{1}(p)} H_{1}^{-1}\left[e_{H_{1}(p)}^{1}, \ldots, e_{H_{1}(p)}^{n-1}\right]\right]\right) \\
& =(G \circ c \circ e)\left(T_{H_{1}(p)}\left(H_{1} \circ i \circ H_{1}^{-1}\right)\left[e_{H_{1}(p)}^{1}, \ldots, e_{H_{1}(p)}^{n-1}\right]\right) \\
& =(G \circ c)\left[e^{1}, \ldots, e^{n-1}\right] \\
& =\left[e^{1}, \ldots, e^{n-1}\right] \\
& =I
\end{aligned}
$$


whereas computing the same thing for $\bar{I}_{1}$, we get

$$
\begin{aligned}
\bar{I}_{1}(f)(p) & =(G \circ e)\left(T_{p}\left(H_{1} \circ i\right)\left(T_{H_{1}(p)} H_{1}^{-1}\left[e_{H_{1}(j(p))}^{1}, \ldots, e_{H_{1}(j(p))}^{n-1}\right]\right)\right) \\
& =(G \circ e)\left(T_{H_{1}(p)}\left(H_{1} \circ i \circ H_{1}^{-1}\right)\left[e_{H_{1}(j(p))}^{1}, \ldots, e_{H_{1}(j(p))}^{n-1}\right]\right) \\
& =I .
\end{aligned}
$$

Thus the two maps agree on both hemispheres, and the lemma is proved.

We now define three sets of immersions, using the notions of bordism and regular homotopy. Bordism and oriented bordism of immersions are defined in [Wel66]. Essentially, two immersions of $k$ manifolds in $n$-space are bordant if there is an immersion of a $k+1$ manifold-with-boundary in $R^{n} \times[0,1]$ whose boundary consists of the original immersions.

DEFINITION. $I(n, k)$ is the set of bordism classes of immersions of $n$-manifolds in $R^{n+k}$. $\mathrm{SI}(n, k)$ is the set of oriented bordism classes of immersions of oriented $n$-manifolds in $R^{n+k}$.

DEFINITION. $\operatorname{Imm}(n, k)$ is the set of regular homotopy classes of immersions of $S^{n}$ in $R^{n+k}$.

REMARK. Both $I(n, k)$ and $\mathrm{SI}(n, k)$ are groups under the operation of "disjoint union of immersions." The inverse of an immersion in $I(n, k)$ is gotten by reflecting through a hyperplane (see [Wel66]). That this is not the inverse in $\operatorname{SI}(n, k)$ will become apparent (see Lemma 2.5 and the discussion at the start of $\S 4)$.

REMARK. Smale's theorem gives a correspondence of a set, namely $\operatorname{Imm}\left(S^{k}, R^{n}\right)$, with a group, $\pi_{k}\left(V_{n, k}\right)$, thus putting a group structure on the set. The following definition of connect-sum of immersions gives a geometric operation on $\operatorname{Imm}\left(S^{k}, R^{n}\right)$. The theorem following it shows that this geometric operation corresponds to the group operation in $\pi_{k}\left(V_{n, k}\right)$.

DEFINITION (oriented connect sum of immersions). Given two good immersions $f, g: S^{k} \rightarrow R^{n}, f$ is regularly homotopic to an immersion $\bar{f}$ agreeing with the standard embedding on all except a disk the northern half of the eastern hemisphere, (i.e., $x_{k+1}>0, x_{k}>0$ ). Similarly $g$ is regularly homotopic to $\bar{g}$ which agrees with the standard embedding on all except the northern half of the western hemisphere $x_{k+1}>0, x_{k}<0$. (See Figure 1.4.) 

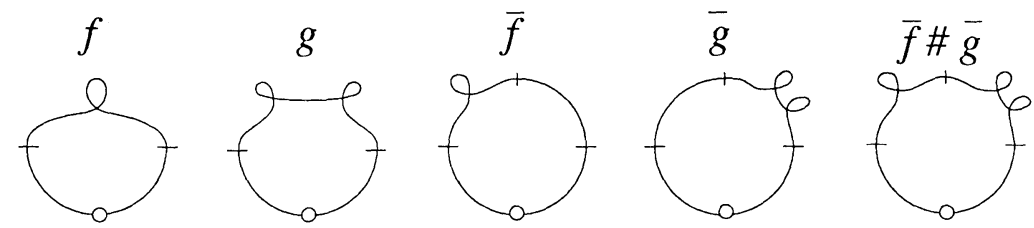

FIGURE 1.4
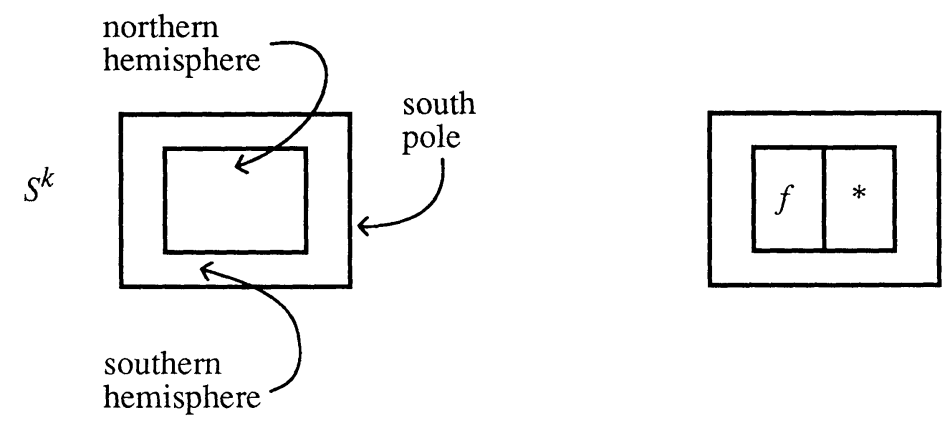

FIGURE 1.5

We now define the oriented-connect-sum (or just connect-sum) of (the regular homotopy classes of) $f$ and $g, f \# g$, to be the immersion

$$
(f \# g)(x)= \begin{cases}\bar{f}(x), & x \in \text { Northeast, } \\ \bar{g}(x), & x \in \text { Northwest }, \\ \bar{f}(x) \text { or } \bar{g}(x), & x \in \text { South. }\end{cases}
$$

REMARK. The immersion $f \# g$ is not really well-defined, for it depends on the choice of $\bar{f}$ and $\bar{g}$. Its regular homotopy class is welldefined however.

THEOREM 1.4. If $f, g: S^{k} \rightarrow R^{n}$ are good immersions, then $I(f \# g)$ $=I(f)+I(g)$ in $\pi_{k}\left(V_{n, k}\right)$.

Proof. By pictures. $S^{k}$ will be drawn as a cube with boundary identified to a point, this point being the south pole. The northern hemisphere is the interior of a concentric cube, and the southern hemisphere the exterior of that cube.

Note that $\bar{f}=\bar{g}$ on the southern hemisphere.

We surround $\bar{f}$ and $\bar{g}$ by the homotopy $H$ used in the proof of Lemma 1.3 to get new maps in $S^{k}$ that send $e^{1}, \ldots, e^{k}$ to $e^{1}, \ldots, e^{k}$ wherever they agree with the standard embedding on $S_{+}^{k}$. Let $*$ denote the frame $\left[e^{1}, \ldots, e^{k}\right]$ in $R^{n}$. The map $I(\bar{f}): S^{k} \rightarrow V_{n, k}$ is depicted schematically in Figure 1.5; the maps $I(\bar{g})$ and $I(f \# g)$ are shown in Figure 1.6. 

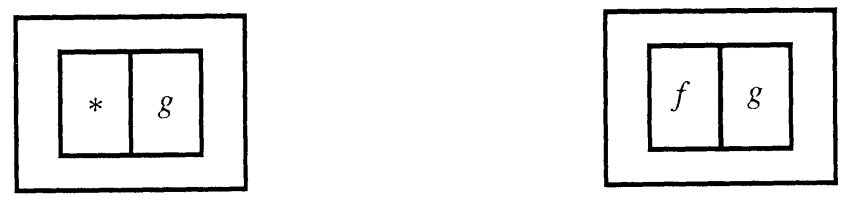

Figure 1.6

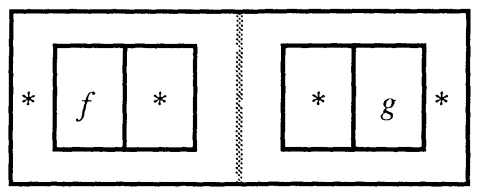

FIGURE 1.7

Note that the homotopy product of $I(\bar{f})$ and $I(\bar{g})$ is gotten by adjoining the pictures for $I(\bar{f})$ and $I(\bar{g})$ along a face, and can thus be shown as in Figure 1.7. This is evidently homotopic to $I(f \# g)$.

REMARK. The constraint that an immersion be good ensures that the south pole is taken to a particular frame in $V_{n, k}$, i.e., ensures that basepoints are preserved so that the elements of the homotopy groups all have the same basepoint. But if $\pi_{1}(X)$ acts trivially on $\pi_{n}(X)$, then basepoints are irrelevant in $\pi_{n}(X)$. This happens in particular for $V_{n, k}$ when $n>k+1$ (since $\pi_{1}$ is then zero, see [Ste51, $\S 25.6]$ ). Also, if $n=k+1$, our computation yields an element not of $\pi_{n-1}\left(V_{n, n-1}\right)$ but of $\pi_{n-1}(\mathrm{SO}(n))$. Now $\mathrm{SO}(n)$, being a topological group, is $n$-simple (i.e., has trivial $\pi_{1}$ action on $\pi_{n}$ ) for every $n$ (see [Ste51, 25.6, 16.6]). Thus we may expand the definition of $I$ to take regular homotopy classes of (not necessarily good) immersions to elements of homotopy groups of either Stieffel manifolds or special orthogonal groups.

Nonetheless, it is important to restrict our attention to good immersions when we apply Smale's theorem to compute $\pi_{1}$ of the space of immersions of $S^{k}$ in $R^{n}$. For example, when $k=2$ and $n=1$, a generator for the fundamental group of the space of all immersions is given by spinning the standard embedding of the sphere on its axis. This is one of two generators. The second is also a generator of the fundamental group of the space of good immersions, and is gotten by everting the sphere twice (see [MB81]).

In the "free homotopy" setting (i.e., no goodness constraint) Figure 1.8 should make it clear that the oriented connect sum of immersions, as just defined, is regularly homotopic to the immersion one gets by 

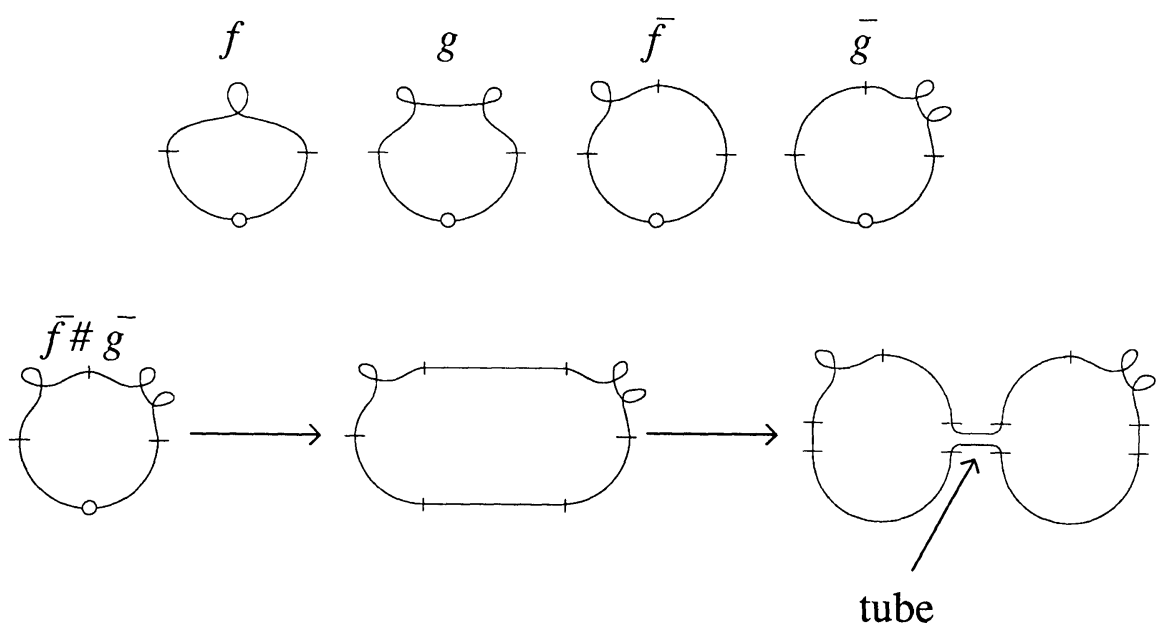

FIGURE 1.8

"tubing together the images of $f$ and $g$. . It is important that the tube be oriented properly.

DEFINITION (tube-connect-sum of immersions). If $f$ and $g$ are immersions of $S^{k}$ in $R^{n}$, the tube-connect-sum of $f$ and $g, f \nvdash g$, is constructed as follows.

Let $p$ be a point of $S^{k}$ and $U$ be a disk about $p$ so small that $f|U, g| U$ are embeddings, and let $\gamma: I \rightarrow R^{n}$ be a smooth curve from $f(p)$ to $g(p)$, such that $\gamma^{\prime}(0)$ is in the normal bundle of $f(U)$ at $f(p)$, and similarly for $\gamma^{\prime}(1)$. Furthermore, if $k=n-1$, let $\gamma^{\prime}(0)$ be the outward normal to $f(U)$ at $f(p)$ and $\gamma^{\prime}(1)$ be the inward normal to $g(U)$ at $g(p)$.

The normal bundle to $\gamma$ in $f(U)$ and $g(U)$ is a trivial $k$-disk bundle over the ends of $\gamma$. The normal bundle to $\gamma$ in $R^{n}$ is a trivial $(n-1)$-disk bundle. The $k$-disk bundle over the ends extends to a $k$-disk sub-bundle of the $(n-1)$-disk bundle (since $\pi_{1} G_{n, k}=0$ unless $k=n-1$, in which case the normality assumption ensures the extension).

Let $\beta: I \times D^{k} \rightarrow R^{n}$ parameterize the image of this $k$-disk bundle in such a way that (considering $I \times S^{k-1}$ as the temperate zone of $\left.S^{k}\right)$

$$
\begin{array}{ll}
\beta(x)=f \circ j_{1}^{-1}(x) & \text { for } x \in \partial \operatorname{arctic}, \\
\beta(x)=g \circ j_{1}^{-1}(x) & \text { for } x \in \partial \text { antarctic, }
\end{array}
$$

where $j_{i}: S^{k}-U \rightarrow S^{k}$ takes $S^{k}-U$ diffeomorphically to the arctic $(i=1)$ or the antarctic $(i=2)$. 
Now define

$$
f \natural g: S^{k} \rightarrow R^{n}: x \rightarrow \begin{cases}f \circ j_{1}^{-1}(x), & x \in \text { arctic, } \\ \beta(x), & x \in \text { temperate zone, } \\ g \circ j_{2}^{-1}(x), & x \in \text { antarctic. }\end{cases}
$$

LEMMA 1.5. If $f$ and $g$ are immersions of a $k$-sphere in $R^{n}$, then $f \# q$ and $f$ hq are regularly homotopic.

Proof. The proof is similar to that of Lemma 1.2, and consists of adjusting the domains to make the two maps agree. We leave the details to the reader.

Henceforth we will use $f \# g$ to denote either oriented- or tubeconnect-sum.

DEFINITION. If $M^{n}$ is a smooth, closed, oriented manifold and $f: M \rightarrow R^{n+1}$ is an immersion, the normal degree of $f, \nu(f)$, is the degree of the Gauss map of $f$. The Gauss map is defined by taking, at each point $p$ of $M$, an oriented frame $\left[v_{p}^{1}, \ldots, v_{p}^{n}\right]$, and considering

$$
n(p)=d f_{p} v_{p}^{1} \times \cdots \times d f_{p} v_{p}^{n} \in T_{f([p])} R^{n+1}
$$

(the vectors $v_{p}^{i}$ need not vary continuously with $p$ ). After normalizing and forgetting basepoint, $n(p)$ lies in $S^{n}$ and varies smoothly with $p$. The map $n: M \rightarrow S^{n}$ is the Gauss map.

2. Regular homotopy classes of immersions of $S^{3}$ in $R^{4}$. This section is devoted to developing tools with which to compute the immersion numbers of a 3-sphere immersed in $R^{4}$, and exhibiting a pair of generators for this group (under the operation of oriented connect sum).

Recall that $\pi_{3} \mathrm{SO}(4)=\pi_{3} S^{3}=Z \oplus Z$, with generators $\sigma$ and $\rho$ (see [Ste51, 22.7]). If $f: S^{3} \rightarrow R^{4}$ is an immersion, then $I[f]$ is in $\pi_{3} \mathrm{SO}(4)$, so $I[f]=\left(I_{1}(f), I_{2}(f)\right) \in Z \oplus Z$. Since $I[f] \in \pi_{3} \operatorname{SO}(4)$, we use $I[f]$ to denote a map from $S^{3}$ to $\mathrm{SO}(4)$ (i.e., a particular representative of the homotopy class of $I[f]$ ). In the same way, we let $I_{1}(f)$ denote a map from $S^{3}$ to $S^{3}$.

LEMMA 2.1. The standard embedding of $S^{3}$ in $R^{4}$ has immersion invariants $(0,0) \in \pi_{3} \mathrm{SO}(4)$.

Proof. The differential applied to the constant frame $\left[e^{1}, \ldots, e^{4}\right]$ is the constant map $S^{3} \rightarrow I \in \mathrm{SO}(4)$. 
LEMMA 2.2. The first immersion number, $I_{1}(f)$, is related to the normal degree, $\nu(f)$, by

$$
I_{1}(f)=\nu(f)-1
$$

Proof. From the definitions,

$$
\begin{gathered}
I_{1}(f)(q)=T_{q} f\left(e_{q}^{1}\right), \\
\nu(f)(q)=T_{q} f\left(q_{q}\right),
\end{gathered}
$$

where $q$ is a point of $S^{3}, e_{q}^{1}$ is the vector $e_{1} \in R^{4}$, considered as an element of the tangent-plus-normal bundle of $S^{3}$ at $q$, and $q_{q}$ denotes the vector from 0 to $q$ in $R^{4}$, considered as an element of the same fiber.

Let $L(q)$ denote left multiplication by the quaternion $q$ acting on $R^{4}$, or on any tangent plane to $R^{4}$. Then at each point $q \in S^{3}$, $L(q): T_{q} R^{4} \rightarrow T_{q} R^{4}$ takes $e_{q}^{1}$ to the normal vector to $S^{3}$ at $q$, namely $q_{q}$. Thus $q_{q}=L(q) e_{q}^{1}$, so

$$
\nu(f)(q)=T_{q} F \cdot L(q)\left[e_{q}^{1}\right] .
$$

The degree of $T_{q} F \cdot L(q)$ is degree $(T f)+$ degree $(L)=I_{1}(f)+1$, and the lemma is proved.

THEOREM 2.3. The standard (Froissart-Morin) eversion of $S^{2}$ in $R^{3}$ has, as a track, an immersion of $S^{2} \times I$ in $R^{4}$, whose ends are embedded $S^{2} s$. Each of these bounds a 3-ball in $R^{4}$. Capping off the track with these 3-balls gives an immersion $K: S^{3} \rightarrow R^{4}$. Performing the eversion twice and capping off gives an immersion $E: S^{3} \rightarrow R^{4}$. The immersion numbers of $E$ and $K$ are

$$
\begin{aligned}
& I[E]=(0,1), \\
& I[K]=(-1,0),
\end{aligned}
$$

perhaps after reflections in the domain and range.

The proof of this theorem requires several preliminary results.

LEMMA 2.4. The composition of the standard embedding with reflection through the $x$-axis of $R^{4}$, namely $r(x, y, z, w)=x,-y,-z$, $-w$, is an immersion with invariants $(-2,1)$. The same is true when the embedding is composed with reflection through the $y$-z-w-plane, 
$r(x, y, z, w)=(-x, y, z, w)$, and hence for reflection through any plane, since all such reflected embeddings are regularly homotopic.

Proof. To compute this, we extend the reflection through the $x$ axis, $(x, y, z, w) \rightarrow(x,-y,-z,-w)$, to an orientation preserving map of a neighborhood of $S^{3}$ in $R^{4}$. Such a map is quaternionic inversion, $q \rightarrow \bar{q} /\|q\|^{2}$, i.e.,

$$
w: S^{3} \times I \rightarrow R^{4}: q=a+b i+c j+d k \rightarrow \frac{a-b i-c j-d k}{a^{2}+b^{2}+c^{2}+d^{2}} .
$$

The differential of this map is just (after restriction to $S^{2}$ ):

$$
T_{q} w=\left|\begin{array}{cccc}
1-2 a^{2} & -2 a b & -2 a c & -2 a d \\
2 a b & -1+2 b^{2} & 2 b c & 2 b d \\
2 a c & 2 c b & -1+2 c^{2} & 2 c d \\
2 a d & 2 d b & 2 d c & -1+2 d^{2}
\end{array}\right|
$$

The first column, $A^{1}$, represents a map from $S^{3}$ to $S^{3}$, which we write $q \rightarrow A^{1}(q)$; its degree is evidently -2 , since it is simply the map $q \rightarrow-q^{-2}$. To compute the second invariant, we must compute $L\left(A^{1}(q)\right)^{-1} \cdot T_{q} w$ which is in $\mathrm{SO}(3) \subset \mathrm{SO}(4)$. (Here $L(q)$ denotes the map from $R^{4}$ to $R^{4}$ given by left multiplication by the quaternion $q$, or the matrix representation of this map with respect to the standard basis. The superscript of -1 indicates matrix inversion.) The computation yields, after considerable simplification,

$L\left(A^{1}(q)\right)^{-1} \cdot T_{q} w=\left|\begin{array}{cccc}1 & 0 & 0 & 0 \\ 0 & 2 b^{2}+2 a^{2}-1 & 2 b c-2 a d & 2 b d+2 a c \\ 0 & 2 a d+2 b c & 2 c^{2}+2 a^{2}-1 & 2 c d-2 a b \\ 0 & 2 b d-2 a c & 2 c d+2 a b & 2 d^{2}+2 a^{2}-1\end{array}\right|$.

This the generator for $\pi_{3}(\operatorname{SO}(3))$ (see [Ste51, 22.7]).

Finally, the result for reflection in the $y-z-w$-plane follows because reflection through the $x$-axis and reflection in the $y-z-w$-plane are isotopic.

REMARK. One way to check this theorem is with a bordism calculation. The $J$ homomorphism, which takes regular homotopy classes of immersions of $S^{n}$ in $R^{n+1}$ and considers them as bordism classes of immersed $n$-manifolds in $R^{n+1}$, is the same as the classical 7 :homomorphism from $\pi_{n} \mathrm{SO}(n+1) \rightarrow \pi_{n}^{s}$ (R. Lee, communicated through Carter). In the case of $\pi_{3} \mathrm{SO}(4)$ to $\pi_{3}^{S}$, it factors through $\pi_{3} \mathrm{SO}(5)$ by inclusion. Since the reflection of the standard sphere is evidently null-bordant, $J(-2, n)=0$, whatever the right $n$ may be. 
But in this dimension, $J(x, y)=x+2 y \bmod 24$. Since we know $n= \pm 1$, the only possible conclusion is $n=1$.

LEMMA 2.5. If $f: S^{3} \rightarrow R^{4}$ is an immersion and $r: R^{4} \rightarrow R^{4}$ : $(x, y, z, w) \rightarrow(x,-y,-z,-w)$ is reflection, then

$$
I(f \circ r)=-I(f)+(-2,1) .
$$

Proof. The extension, $\bar{f}$, of $f$ used to compute $I(f)$ will not do for computing $I(f \circ r)$ (it is not an orientation preserving extension). Nonetheless, if we let $w: S^{3} \times I \rightarrow S^{3} \times I$ denote quaternionic inversion, then $\bar{f} \circ w$ is an orientation preserving extension of $f \circ r$, since $w=r$ on $S^{3}$, and both $\bar{f}$ and $w$ are orientation preserving maps from subsets of $R^{4}$ to $R^{4}$. To compute $I(f \circ r)$ we need therefore only compute the homotopy class of $T(\bar{f} \circ w)$ in $\pi_{3} \mathrm{SO}(4)$. Since

$$
T_{q}(\bar{f} \circ w)=T_{w(q)} \bar{f} \cdot T_{q} w
$$

and point by point multiplication of matrices is the sum in $\pi_{3} \mathrm{SO}(4)$, we get that $I(f \circ r)$ is the sum of the classes represented by $(T \bar{f}) \circ$ $w$ and $T w$. Now $(T \bar{f}) \circ w$ represents $-I(f)$, since reversing the orientation of $S^{3}$ (which is what $w$ does) changes the sign of an element of $\pi_{3} \mathrm{SO}(4)$, and $T w$ represents $(-2,1)$, by the proof of Lemma 2.2.

The computation of the immersion invariant when a reflection is made in the image rather than the domain is substantially more complicated.

LEMMA 2.6. If $f: S^{3} \rightarrow R^{4}$ is an immersion with invariants $(s, p)$, and $r: R^{4} \rightarrow R$ denotes reflection in the x-axis, then the immersion invariants of $r \circ f$ are given by

$$
I(r \circ f)=(-2-s, 1+p+s) .
$$

Proof. Again the proof relies on choosing the proper extension for $r \circ f$ given an extension, $\bar{f}$, of $f$ to $S^{3} \times I$. In this case, an orientation preserving extension of $r \circ f$ is given by

$$
\overline{r \circ f}=r \circ \bar{f} \circ u \text {, }
$$

where $u: R^{4} \rightarrow R^{4}: q \rightarrow q /\|q\|^{2}$. In coordinates,

$$
u(a, b, c, d)=\frac{1}{a^{2}+b^{2}+c^{2}+d^{2}}(a, b, c, d) .
$$


For convenience, let $J$ denote the matrix

$$
J=\left|\begin{array}{rrrr}
1 & 0 & 0 & 0 \\
0 & -1 & 0 & 0 \\
0 & 0 & -1 & 0 \\
0 & 0 & 0 & -1
\end{array}\right| \text {. }
$$

Note that $J^{2}=1$, the identity matrix, and $J=d r_{x}$ for every $x$. Now the differential of $\overline{r \circ f}$ can be computed as follows:

$$
\begin{aligned}
T_{q}(\overline{r \circ f}) & =T_{\bar{f}(u(x))} r \cdot T_{u(x)} \bar{f} \cdot T_{x} u \\
& =T_{\bar{f}(x)} r \cdot T_{x} \bar{f} \cdot T_{x} u \quad \text { for } x \in S^{3} \\
& =J T_{x} \bar{f} J T_{x} w
\end{aligned}
$$

where $w: R^{4} \rightarrow R^{4}: q \rightarrow \bar{q} /\|q\|^{2}$ is quaternionic inversion, so that $u=r \circ w$. To compute which homotopy class this represents in $\pi_{3} \mathrm{SO}(4)$, we need only observe that $T w: S^{2} \rightarrow \mathrm{SO}(4)$ represents the class $(-2,1)$ by the proof of Lemma 2.4 , and then we must compute the class represented by $J \cdot T \bar{f} \cdot J$. Recall that the immersion invariants of $f$ are $(s, p) . T \bar{f}$ must be homotopic to a product of left and right quaternion multiplications (since these generate $\pi_{3} \mathrm{SO}(4)$ - see [Ste51, §22.7]). In particular, using the specific generators $q \rightarrow L(q)$ and $q \rightarrow R(q)$, where $R(q)$ denotes right multiplication by $q$, to write the generators $\sigma$ and $\rho(\sigma$ is the class $(1,0)$, while $\rho$ is $(0,1))$, we get

$$
T_{q} \bar{f}=L\left(q^{s}\right) L\left(q^{p}\right) R\left(q^{-p}\right) .
$$

Observing that the matrices for $L(q)$ and $R(q)$ are

and

$$
L(q)=\left|\begin{array}{rrrr}
a & -b & -c & -d \\
b & a & -d & c \\
c & d & a & -b \\
d & -c & b & a
\end{array}\right|
$$

$$
R(q)=\left|\begin{array}{rrrr}
a & -b & -c & -d \\
b & a & d & -c \\
c & -d & a & b \\
d & c & -b & a
\end{array}\right|
$$

we can see that $J \cdot L(q) \cdot J=R(q)^{t}=R\left(q^{-1}\right)$. Applying this to the formula for $T \bar{f}$, we get

$$
\begin{aligned}
J \cdot T_{q} \bar{f} \cdot & =J \cdot L\left(q^{s}\right) \cdot L\left(q^{p}\right) \cdot R\left(q^{-p}\right) \cdot J \\
& =J \cdot L\left(q^{s}\right) \cdot J \cdot J \cdot L\left(q^{p}\right) \cdot J \cdot J \cdot R\left(q^{-p}\right) \cdot J \\
& =R\left(q^{-s}\right) \cdot R\left(q^{-p}\right) \cdot L\left(q^{p}\right) \\
& =L\left(q^{-s}\right) \cdot L\left(q^{p+s}\right) \cdot R\left(q^{-(p+s)}\right) .
\end{aligned}
$$


Thus the total immersion invariant for $r \circ f$ is $(-s, p+s)$ added to $(-2,1)$, which proves the lemma.

Given a homotopy, $H$, of maps from $S_{-}^{2}$ to any space, $X$, we may, provided $H$ satisfies certain conditions, define $\eta(H)$ as an element of $\pi_{3} X$ in the following manner. First of all, $H: S_{-}^{2} \times I \rightarrow X$ must satisfy the condition $H(p, 0)=H(p, 1)$ for all $p \in S_{-}^{2}$ (i.e., $H$ must be a loop of maps), and secondly, $H$ must satisfy $H(p, t)=H\left(p, t^{\prime}\right)$ for all $p \in \partial S_{-}^{2}$ and all $t, t^{\prime} \in I$.

In this case, there is a map defined on the quotient of $S_{-}^{2} \times I$ by these identifications, which is homeomorphic to $S^{3}$. This quotient map is called $\eta(H)$.

LEMMA 2.7. If two homotopies, $H, K: S_{-}^{2} \times I \rightarrow X$ are homotopic by a homotopy which respects the identifications used in defining $\eta$, then $\eta(H)=\eta(K)$.

Lemma 2.8. The map $i: V_{3,2} \rightarrow V_{3,3}:\left[v^{1}, v^{2}\right] \rightarrow\left[v^{1}, v^{2}, v^{1} \times v^{2}\right]$ induces an isomorphism on $\pi_{3}$.

Proof. The fiber of this map is $S^{0}$, so the result follows from the homotopy sequence of a fibration.

Banchoff and Max prove that, if $J: S_{-}^{2} \times I \rightarrow R^{3}$ is twice the Froissart-Morin eversion (restricted to the southern hemisphere), and we define $d J(p, t)=T_{p} J_{t}\left[v_{p}^{1}, v_{p}^{2}\right]$, where $\left[v_{p}^{1}, v_{p}^{2}\right]$ is the frame on the southern hemisphere induced by stereographic projection of the standard frame on $R^{2}$ then $\eta(d J)$ is a generator of $\pi_{3} V_{3,2}$ (see [MB81]).

LEMMA 2.9. Let $\widetilde{J}$ denote an extension of $J$ to an orientation preserving immersion of a neighborhood of $S_{-}^{2}$ (for each $t$ ). If we define $d \tilde{J}$ by

$$
d \widetilde{J}(p, t)=T_{p} \widetilde{J}_{t}\left(v_{p}^{1}, v_{p}^{2}, v_{p}^{1} \times v_{p}^{2}\right),
$$

then $\eta(d \widetilde{J})=i \eta(d J)$.

Proof. Given any two oriented frames in $R^{3}$ with the same first two vectors, say $\left[a^{1}, a^{2}, a^{3}\right]$ and $\left[a^{1}, a^{2}, b^{3}\right]$, there is a natural deformation from one to the other through oriented frames: $\left[a^{1}, a^{2}\right.$, $\left.s \cdot a^{2}+(1-s) \cdot b^{3}\right]$. Applying this deformation pointwise to the frames $i(d J)$ and $d \widetilde{J}$ proves the lemma, since $i$ and $\eta$ commute. 
We may therefore conclude that for the regular homotopy $J, d \widetilde{J}$ is a generator of $\pi_{3} V_{3,3}$.

We now alter the choice of the frame that is pushed forward. Since any two (oriented) framings of the trivial 3-plane bundle over the lower hemisphere (i.e., sections of the frame bundle of this bundle) are homotopic (the lower hemisphere is contractible), we pick a homotopy $H$ from the framing $\left[v_{p}^{1}, v_{p}^{2}, v_{p}^{1} \times v_{p}^{2}\right]$ to $\left[e^{1}, e^{2}, e^{3}\right]$ (the constant frame consisting of the standard basis vectors). If we define $U-s$ : $S_{-}^{2} \times I \rightarrow V_{3,3}:(p, t) \rightarrow T_{p} \widetilde{J}_{t} H(s)$ then we see that $d \widetilde{J}$ is just $U_{0}$. Furthermore, $\eta\left(U_{s}\right)$ is independent of $s$ (since each stage $U-s$ respects the identifications in the definitions of $\eta)$. Hence $\eta\left(U_{1}\right)$ generates $\pi_{3} V_{3,3}$. We restate this observation in the following lemma:

LEMMA 2.10. If $J$ is $F$ (the Froissart-Morin eversion) followed by $r F$, and $\widetilde{J}_{t}$ is (for each stage of the homotopy) an extension of $J_{t}$ to an orientation preserving immersion of a neighborhood of $S_{-}^{2}$ in $R^{3}$, and

$$
Q: S_{-}^{2} \times I \rightarrow V_{3,3}:(p, t) \rightarrow T_{p} J_{t}\left[e^{1}, e^{2}, e^{3}\right]
$$

then $\eta(Q)$ generates $\pi_{3} V_{3,3}$.

We now use this observation to compute the immersion invariant of the immersion of $S^{3}$ in $R^{4}$ gotten by capping off the track of the doubled Froissart-Morin eversion. To do this, we identify $S^{3}$ once and for all as the union of three parts: a lower hemisphere, consisting of those points of a unit sphere with center $(0,0,0,0)$ whose fourth coordinate is non-positive; a center cylinder, $S^{2} \times I$, with radius 1 and the segment from $(0,0,0,0)$ to $(0,0,0,1)$ as its axis; and an upper unit hemisphere, with center at $(0,0,0,1)$, and whose points all have further coordinate greater than or equal to one (see Figure 2.1).

We immerse this sphere into $R^{4}$ in three parts as well: the lower hemisphere is immersed by the identity; the center cylinder is immersed by the track of the regular homotopy $J$; and the upper hemisphere is immersed by the identity as well. This is the immersion we call $E$.

To evaluate the immersion invariant, we extend this to an orientation preserving immersion of a neighborhood of $S^{3}$. We then push forward the standard frame at each point by the differential of this extended immersion to get an element of $\pi_{3} V_{4,4}=\pi_{3} \mathrm{SO}(4)$, which is the immersion invariant. 


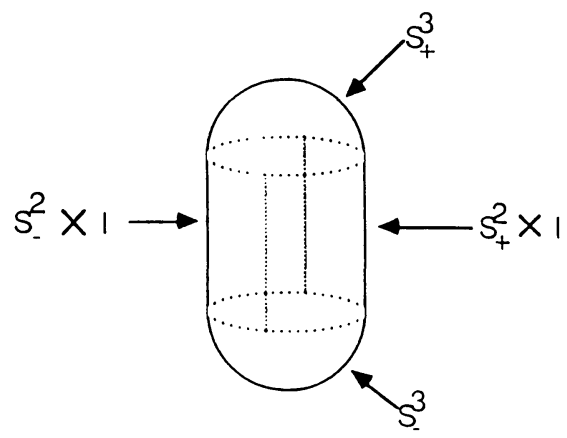

FIGURE 2.1

Let us compute this invariant piece-by-piece as well. On both the northern and southern hemispheres, the original immersion is the identity, so that we may extend it to be the identity on a tubular neighborhood of these sets. Once we have performed this extension, the differential of the immersion is the identity on these regions, hence the standard frame is pushed forward to the standard frame (the basepoint of $\left.V_{4,4}\right)$.

Similarly, since the northern hemisphere of $S^{2}, S_{+}^{2}$, is left fixed by each stage of $J$, we see that $S_{+}^{2} \times I$ is also immersed by the identity in the original immersion. By the same argument, we may therefore extend it so that the standard frame is pushed forward to the standard frame.

Finally on the southern hemisphere of $S^{2}$, we have computed the value of the differential of $J$ on the standard frame of $R^{2}$. The new (extended) immersion can be defined on $S_{-}^{2} \times I$ by

$$
K(p, t)=\left(\widetilde{J}_{t}(p), t\right),
$$

where $\widetilde{J}$ is the orientation preserving extension of $J$ used above.

The differential of this extended immersion is therefore the direct product of the differential of $\widetilde{J}$ and the 1-by-1 identity.

Hence the immersion invariant for the immersed $S^{3}$ is just $\alpha \eta(d \widetilde{J})$, where $\alpha: V_{3,3} \rightarrow V_{4,4}:\left[v^{1}, v^{2}, v^{3}\right] \rightarrow\left[v^{1}, v^{2}, v^{3}, e^{4}\right]$ and the $v^{i} s$ on the right hand side are the inclusions of the corresponding vectors from the left into $R^{4}$.

This is nearly the generator $\pm \rho$ of $\pi_{3} V_{4,4}$, except it is in the wrong corner; we correct this with the following:

LEMMA 2.11. If $q \rightarrow P(q)$ is a map of $S^{3}$ to $\mathrm{SO}(3)$, then $q \rightarrow S_{1}(q)$ : and $q \rightarrow S_{2}(q)$ are homotopic maps from $S^{3}$ to $\mathrm{SO}(4)$, where $S_{1}(q)$ 
and $S_{2}(q)$ are defined by

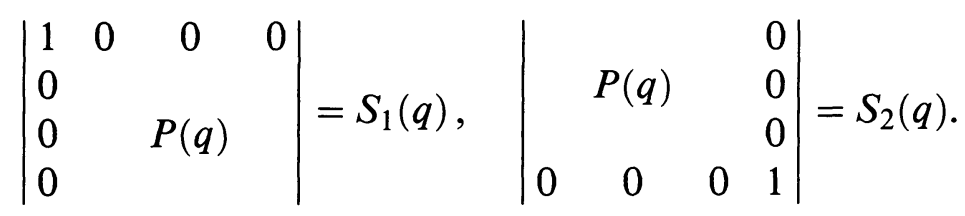

Proof. Let $M(t)$ be a path in $\mathrm{SO}(4)$ with $M(0)=I$ and

$$
M(1)=\left|\begin{array}{rrrr}
0 & 0 & 0 & -1 \\
1 & 0 & 0 & 0 \\
0 & 1 & 0 & 0 \\
0 & 0 & 1 & 0
\end{array}\right|
$$

Then $M(0) \cdot S_{1}(q) \cdot M(0)^{t}=S_{1}(q)$ and $M(1) \cdot S_{1}(q) \cdot M(1)^{t}=$ $S_{2}(q)$. Thus $H(q, s)=M(s) \cdot S_{1}(q) \cdot M(s)^{t}$ provides the required homotopy.

Thus the immersion invariants of $E$ must be $(0,1)$ or $(0,-1)$. If the invariant is $(0,1)$, we may alter $E$ by a reflection in both the domain and range. Lemmas 2.5 and 2.6 then let us compute

$$
I\left(E^{\prime}\right)=-(-2,-0,1+0+-1)+(-2,1)=(0,1) .
$$

Thus we have:

THEOREM 2.12. For the immersion $E$ of $S^{3}$ in $R^{4}$, the invariants are $(0,1) \in \pi_{3} \mathrm{SO}(4)=Z \oplus Z$. (Perhaps after reflections in both domain and range).

THEOREM 2.13. For the immersion $K$ of $S^{3}$ in $R^{4}$, the invariants are $(-1,0)$. (Perhaps after a reflection in the domain.)

Proof. The proof depends on the results proved in the beginning of this section. It is evident that if $r_{1}$ is reflection in the $y-z-w$-plane of $R^{4}$ and $r_{2}$ is reflection in the $x-y$-w-plane of $R^{4}$, then

$$
E=\left(K r_{2} K\right) \cdot r_{1}
$$

(recall '\#' denotes oriented connect sum of immersions).

The computation of $I(K)$ from this formula goes as follows. First of all, the normal degree of $K$ is zero, since the normal vector to $K$ never points in the $e^{4}$ direction (it does point sometimes in the $-e^{4}$ direction), so $I(K)=(-1, n)$ for some $n$. Thus $I\left(r_{2} K\right)=(-1, n)$ 
as well, by Lemma 5.6. Finally, using the fact that $I(f \circ r)=(-2,1)-$ $I(f)$, we get

$$
\begin{aligned}
I(E)=(0,1) & =(-2,1)-\{(-1, n)+(-1, n)\} \\
& =(-2,1)+(2,-2 n) \\
& =(0,1-2 n)
\end{aligned}
$$

so that $1=1-2 n$, so $n=0$.

In the event that $I(E)=(0,-1)$ we get $I(K)=(-1,-1)$. But then composing with a reflection in the domain gives $K^{\prime}$ with $I\left(K^{\prime}\right)=$ $(-1,0)$, by Lemma 2.5 .

3. Immersions in $R^{4}$ that bound: invariants and constructions. When an immersed $S^{3}$ in $R^{4}$ bounds an immersion of an orientable 4manifold in $R^{4}$, the immersion invariants are related to the characteristic classes of the 4-manifold. We compute this relationship in Theorem 3.1.

THEOREM 3.1. If $M$ is a closed, compact, oriented 4-manifold, $U$ is a 4-ball in $M, M_{0}=M-U$, and $f: M_{0} \rightarrow R^{4}$ is an orientation preserving immersion, then $f$ restricted to the boundary of $M_{0}$ is an immersion of $S^{3}$, and

$$
I\left(f \mid \partial M_{0}\right)=\left(\chi(M)-2,-\frac{1}{4} p_{1}(M)-\frac{1}{2} \chi(M)+1\right),
$$

where $\chi(M)$ is the Euler characteristic of $M$ and $p_{1}(M)$ is the Pontriagin number of $M$.

Proof. The proof comes in three steps:

(1) To compute $I\left(f \mid \partial M_{0}\right)$, we compare a pushed-forward frame from $\partial M_{0}$ with the constant frame on $R^{4}$.

(2) To compute the clutching function for $T M$ (hence compute $\chi(M)$ and $p_{1}(M)$ ), we compare a framing of $M_{0}$ (we use the pullback of the constant frame on $R^{4}$ ) with the constant frame on $U$.

(3) We check orientations and some facts about conjugation in homotopy to conclude that (1) and (2) are related.

Step 0 . The Gram-Schmidt process, $G: \mathrm{SL}(4) \rightarrow \mathrm{SO}(4)$ induces an isomorphism in homotopy, so we work in $\mathrm{SL}(4)$ rather than $\mathrm{SO}(4)$ when it is more convenient.

Step 1. Let $\left[\varepsilon^{1}, \ldots, \varepsilon^{4}\right]$ be an oriented frame on $U$ and $\left[e^{1}\right.$, $\left.\ldots, e^{4}\right]$ be the standard frame on $R^{4}$. Then for each $p \in \partial U$, 
$\left[T_{p} f\left(\varepsilon^{1}\right), \ldots, T_{p} f\left(\varepsilon^{4}\right)\right]$ is a frame at $f(p)$. Denote this frame (or element of SL(4)) by $s(p)$. (One gets an element of SL(4) by writing the vectors $T_{p} f\left(\varepsilon^{i}\right)$ in terms of the standard basis.) Loosely (ignoring the Gram-Schmidt process), $[s]=I(f) \in \pi_{3} \operatorname{SL}(4)$.

Step 2. Letting $f^{*}(p)$ denote the inverse map to $T_{p} f$, we have a frame $\left[f^{*}(p) e^{1}, \ldots, f^{*}(p) e^{4} ; p\right]$ of $T_{p} M_{0}$ for each $p \in M_{0}$. Since $f$ is orientation preserving, this frame gives the same orientation as $\left[\varepsilon^{1}, \ldots, \varepsilon^{4}\right]$ on $\partial U$. Hence there is, for each $p \in \partial U$, an element of $\mathrm{SL}(4)$ (we call it $c(p)$ ) such that

$$
\left[\varepsilon^{1}, \ldots, \varepsilon^{4}\right]=c(p) \cdot\left[f^{*}(p) e^{1}, \ldots, f^{*}(p) e^{4}\right] .
$$

(All vectors are written with respect to the basis $\left[\varepsilon^{1}, \ldots, \varepsilon^{4}\right]$. )

This clutching function, $c: \partial U \rightarrow \mathrm{SL}(4)$ is related to the characteristic classes $\chi$ and $p_{1}$. First, suppose that we have applied the Gram-Schmidt process, so that $c: \partial U \rightarrow \mathrm{SO}(4)$.

Now let $k: S^{3} \rightarrow \partial U$ be an orientation preserving diffeomorphism, and let $r: S^{3} \rightarrow S^{3}$ be quaternionic inversion. Then $k \circ r: S^{3} \rightarrow \partial U$ is orientation preserving and

$$
\bar{c}=c \circ k \circ r: S^{3} \rightarrow \mathrm{SO}(4)
$$

is related to the characteristic classes of $M$.

First of all, composing with projection of a frame to its first vector, $\mathrm{SO}(4) \rightarrow S^{3}:\left[A^{1}, \ldots, A^{4}\right] \rightarrow A^{1}$, gives a map $\pi_{1} \circ \bar{c}: S^{3} \rightarrow S^{3}$, whose degree is the Euler characteristic of $M_{0}$ (since its degree is precisely the obstruction to extending the vector field $f^{*}(p) e^{1}$ over the last disk, $U$, of $M$ ).

Furthermore, composing with $i: \mathrm{SO}(4) \rightarrow \mathrm{SO}(5)$, the map which takes a 4-by-4 matrix and makes it 5-by-5 by adding 1 in the upper lefthand corner, gives an element of $\pi_{3} \mathrm{SO}(5)$. This is just $-\frac{1}{2} p_{1}(M)$ (see [KM58]). Furthermore, $i: \pi_{3} \mathrm{SO}(4) \rightarrow \pi_{3} \mathrm{SO}(5):(a, b) \rightarrow(a+2 b)$, where we are using, as generators of $\pi_{3} \mathrm{SO}(4)$ and $\pi_{3} \mathrm{SO}(5)$, the standard elements $\sigma$ and $\rho$, and $i \sigma$, respectively. (See [Ste51, $\S 22.7$, 23.6].)

Thus, if we write $(a, b) \in Z \oplus Z=\pi_{3} S^{3}+\pi_{3} \mathrm{SO}(3)=\pi_{3} \mathrm{SO}(4)$ for $I(f)$, we get

and

$$
i(a, b)=a+2 b=-\frac{1}{2} p_{1}(M)
$$

$$
\begin{aligned}
a & =\chi(M), \text { so } \\
-2 b & =\chi(M)+\frac{1}{2} p_{1}(M), \text { and } \\
b & =-\frac{1}{2} \chi(M)-\frac{1}{4} p_{1}(M) .
\end{aligned}
$$


Hence $\bar{c}$ represents the element $\left(\chi(M),-\frac{1}{2} \chi(M)-\frac{1}{4} p_{1}(M)\right)$ in $\pi_{3} \mathrm{SO}(4)$.

Step 3. Evidently $s$ and $\bar{c}$ are related. Letting $j(p)$ denote the matrix of $T_{p} f$ with respect to the bases $\left[\varepsilon^{1}, \ldots, \varepsilon^{4}\right]$ and $\left[e^{1}, \ldots, e^{4}\right]$, we have $c(p)=j(p)^{-1} \cdot s(p) \cdot j(p)$. Letting $\bar{s}=s \circ k$, we get that

$$
\begin{aligned}
{[\bar{c}] } & =-[\bar{c} \circ r] \\
& =-[c \circ k \circ r \circ r] \\
& =-[c \circ k] \\
& =-\left[(j \circ k)^{-1}\right]-[s \circ k]-[j \circ k] \\
& =-[s \circ k] \\
& =-[\bar{s}] .
\end{aligned}
$$

Thus $[\bar{c}]=-[\bar{s}]$ is $I(f \mid \partial U)$, and we wish to find $I\left(f \mid \partial M_{0}\right)$. Since this is just the oppositely oriented immersion, we use Lemma 2.5 to get $I\left(f \mid \partial M_{0}\right)=(-2,1)-I(f \mid \partial U)=(-2,1)+[c]=(-2,1)+$ $\left(\chi(M),-\frac{1}{2} \chi(M)-\frac{1}{4} p_{1}(M)\right)$, which proves the theorem.

REMARK. It is clear that this theorem works in more generality: the immersion invariant of a codimension one immersion of $S^{n}$ is related to the clutching function of the tangent bundle of an immersed $(n+1)$-manifold which it bounds. If one chooses the usual orientation convention, as we have done, the clutching function must be altered by the immersion invariant of the reflected sphere $((2,-1)$ in this case $)$ to get the immersion invariant we desire. If the boundary immersion is given the opposite orientation, then the clutching function and immersion invariant are related directly. The only remaining step, for the general case of the $n$-sphere in codimension one, is to identify the relation of the clutching function of an almost parallelizable manifold to the characteristic classes of the manifold.

4. Groups of low dimensional immersions. The lowest dimensional immersion groups are $I(0, k)=\mathrm{SI}(0, k)=Z / 2 Z$ (generated by a map of a point to $\left.R^{k}\right)$, and $\operatorname{Imm}(0, k)=0($ all $k) ; I(1,1)=$ $\operatorname{SI}(1,1)=Z / 2 Z, \operatorname{Imm}(1,1)=Z$, all generated by the Figure 8 ; $I(1, k)=\operatorname{SI}(1, k)=\operatorname{Imm}(1, k)=0 \quad(k \geq 2)$.

The groups $I(2,1)=Z / 8 Z$ and $\operatorname{SI}(2,1)=Z / 2 Z$ have been extensively studied (see [Bro70], [Car86], [HH85], [Ban74], [Ecc79], [Ecc80]). $\operatorname{Imm}(2,1)=0$, by Smale's theorem, with the remarkable corollary that a sphere may be turned inside out. 
For 3-manifolds, the following groups are computed in [Wel66] and [Ste51]:

$$
\begin{array}{ll}
I(3,1)=Z / 2 Z, & \operatorname{SI}(3,1)=Z / 24 Z, \\
I(3,2)=Z / 2 Z, & \operatorname{SI}(3,2)=Z / 2 Z, \quad \operatorname{Imm}(3,2)=Z / 2 Z .
\end{array}
$$

In this section we give generators for each of these, and geometric invariants that help determine the class of an immersion in these groups.

The map $\operatorname{Imm}\left(S^{3}, R^{4}\right)=Z \oplus Z$ to $\operatorname{SI}(3,1)$ can be computed as follows (see [Fre78]). The map factors as

$$
\pi_{3} \mathrm{SO}(4) \rightarrow \pi_{3} \mathrm{SO}(5) \rightarrow \cdots \rightarrow \pi_{3} \mathrm{SO} \rightarrow \pi_{3}^{s}\left(S^{1}\right)
$$

where the last map is the classical $J$-homomorphism, which is a surjection. Since $\pi_{3} \mathrm{SO}(n) \rightarrow \pi_{3} \mathrm{SO}(n+1)$ is an isomorphism for $n \geq 5$, we need only compute the map

$$
Z \oplus Z=\pi_{3} \mathrm{SO}(4) \rightarrow \pi_{3} \mathrm{SO}(5)=Z .
$$

According to [Ste51] this is just $(a, b) \rightarrow a+2 b$. Thus the image of the immersion $K$ (the capped eversion) will be $J(K)=1(\bmod 24)$, a generator.

Note that $K$ has a single quadruple point. The number of quadruple points of a generic immersion, taken mod 2, is a bordism invariant, so even in $I(3,1), K$ must represent a non-zero element. But $I(3,1)=Z / 2 Z$, so $K$ is only non-zero class.

Similarly, if we include $K$ into $R^{5}$, we get a new immersion $K^{\prime}$ : $S^{3} \rightarrow R^{5}$. The invariant of $K^{\prime}$ can be computed from the invariant of $K,(1,0)$, by working on the level of homotopy groups. For this purpose we return to Smale's definition of the immersion invariant and think of $I(K)$ as lying in $\pi_{3}\left(V_{4,3}\right)$ rather than $\pi_{3} \mathrm{SO}(4)$. The inclusion of $R^{4}$ induces a map from $V_{4,3}$ to $V_{5,3}$ which appends a zero as the last coordinate of each of the vectors in a frame. On the level of the definitions, since

$$
V_{4,3}=\frac{\mathrm{SO}(4)}{\mathrm{SO}(1)} \quad \text { and } \quad V_{5,3}=\frac{\mathrm{SO}(5)}{\mathrm{SO}(2)}
$$

the map is inclusion of $\mathrm{SO}(4)$ into $\mathrm{SO}(5)$ modulo the inclusion of $\mathrm{SO}(1)$ into $\mathrm{SO}(2)$. For the sake of computing $\pi_{3}, V_{5,3}$ is the same as $\mathrm{SO}(5)$, since $\pi_{3} S^{1}=\pi_{2} S^{1}=0$. The map from $\pi_{3} \mathrm{SO}(4)$ to $\pi_{3} \mathrm{SO}(4)$ (with respect to the generators in Chapter 20 of [Ste51]) is just $(a, b) \rightarrow a+2 b$; hence the invariant of $K^{\prime}$ is just $1+2 \cdot 0=1$. 
Thus $K^{\prime}$ generates $\operatorname{Imm}(3,2)$. We now exhibit an immersion $L: S^{3} \rightarrow R^{5}$ which generates $I(3,2)$, hence conclude that the map $\operatorname{Imm}(3,2) \rightarrow I(3,21)$ is surjective. This then shows that $K^{\prime}$ also generates $I(3,2)$ and $\operatorname{SI}(3,2)$.

The immersion $L$ is defined by

$$
\left|\begin{array}{c}
x \\
w \\
z \\
w
\end{array}\right| \rightarrow\left|\begin{array}{c}
2 x y+w\left(x^{2}-y^{2}\right) \\
x z-w y+x\left(x^{2}+y^{2}\right) \\
-y z-w x+y\left(x^{2}+y^{2}\right) \\
z \\
w
\end{array}\right|
$$

The enthusiastic reader may verify that this does, in fact, define an immersion, with double point set given by

$\left\{d_{\theta}=\left(\beta \cos \theta, \beta \sin \theta, \beta^{2} \cos (\pi-2 \theta), \beta^{2} \sin (\pi-2 \theta)\right): \theta \in[0,2 \pi]\right\}$

where

$$
\beta=\sqrt{(\sqrt{5}-1) / 2}
$$

Note that $L\left(d_{\theta}\right)=L\left(d_{\theta+\pi}\right)$. Thus the double point set, parameterized by $\theta$, is a single circle in the image, double covered by a single circle in the domain. Therefore a neighborhood of the double curve in the image must be a nontrivial $D^{2} \wedge D^{2}$ bundle over $S^{1}$. Theorem 4.1 below shows that $L$ must therefore be a generator of $I(3,2)$.

For a generic immersion of a 3-manifold in $R^{5}$, define the twist of a double curve as follows: a neighborhood of the double curve in the image contains a portion of the image of the 3-manifold. This portion is a $D^{2} \wedge D^{2}$ bundle over the $S^{1}$. The twist is zero if the $D^{2} \wedge D^{2}$ bundle is a product bundle, and is one otherwise. The total twist of the immersion is the sum of the twists along all double curves, taken mod 2. (This definition applies only to generic immersions. To apply it to a non-generic immersion, the immersion must first be perturbed to be generic.)

THEOREM 4.1. Total twist is a bordism invariant for 3-manifolds in $R^{5}$.

Proof. The only events that occur during a generic bordism of a 3-manifold in $R^{5}$ are

(1) Addition of handles (by general position, we may assume the attaching regions for 0-, 1-, and 2-handles all miss the double curves). By turning upside down, we convert 3-handle additions to 0-handle additions, which we may once again assume to miss the double curves. 
(2) Creation of new double curves (or extermination of old ones).

(3) Merger or splitting of double curves (including self-merging), and

(4) Appearance of a triple point (transient).

The last three all occur during regular homotopies as well, and in fact away from levels at which handles are added, a bordism is a regular homotopy. It is known that twisting invariants like this are regular homotopy invariants, but we will give a few details regardless.

It is evident that (1) and (4) have no effect on the triviality of the $D^{2} \wedge D^{2}$ bundle over any double curve in (2), only trivial new bundles are formed (which one sees by looking at a standard model of doublecurve creation), hence the total twist does not change. In case (3), we describe the situation when two distinct curves merge, and leave the remaining cases to the reader.

In a neighborhood of the double curve, the immersion must locally resemble the intersection of 3-planes in $R^{5}$. We call each 3-plane a sheet, although this makes sense only locally.

A neighborhood of a merger must look like Figure 4.1 in the preimage of one sheet of the intersection. The arcs are the preimages of the double curves, and the two parts of the figure show these preimages before and after the merge.

If there is a section of the $D^{2} \wedge D^{2}$ bundle over each sheet in Figure 4.1(a), we may suppose that these sections are in our sheet, and hence locally resemble Figure 4.2(a).

One can evidently convert this to a section of the bundle after the merger, as in Figure 4.2(b).

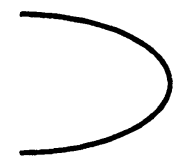

$\mathrm{a}$

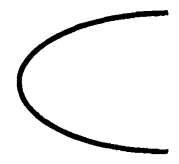

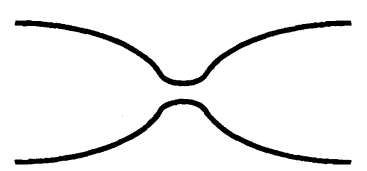

b

FIGURE 4.1

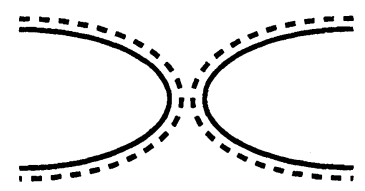

a

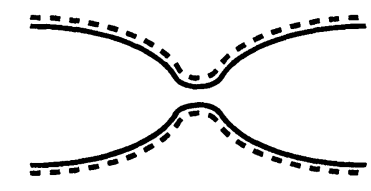

b

FIGURE 4.2 


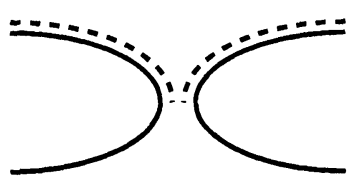

a

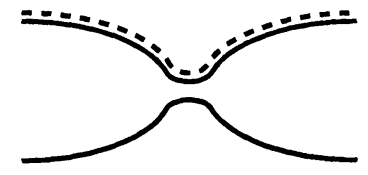

b

FIGURE 4.3

In the event that both components of the double curve before the merger have twist one, a similar procedure works. One simply takes a section of the bundle, as shown in Figure 4.3(a), away from the merger point. The dotted line indicates that the cross section is in this sheet as it approaches the merger point from one side; the absence of the dotted line in the lower half of the figure indicates that it is in the other sheet there. After the merger has taken place, the section can be made continuous, by joining the two dotted lines in Figure 4.3(a), and joining the two dotted lines that appear in the corresponding view of the situation in the other sheet. Thus after the merger the bundle is trivial, and the total twist for these two components is unchanged.

Since there exists an immersion of $S^{3}$ in $R^{5}$ with total twist one, the twist map is onto $Z / 2 Z$. Hence we have proved

THEOREM 4.2. The total twisting along double curves, taken mod 2, is a complete bordism invariant for oriented or unoriented 3-manifolds in $R^{5}$.

Total twist is inadequate as a measure of the regular homotopy class of an immersed $S^{3}$ in $R^{5}$, for there are examples of embedded $S^{3} S$ in $R^{5}$ that are not regularly homotopic to the standard embedding (see [HM85]).

\section{REFERENCES}

[Ban74] T. F. Banchoff, Triple points and surgery of immersed surfaces, Proc. Amer. Math. Soc., 46 (1974), 403-413.

[Bro70] E. H. Brown, Framed manifolds with a fixed point free involution, Michigan Math. J., 23 (1970), 257-260.

[Car86] J. S. Carter, Surgery on codimension one immersions in $R^{n+1}$ : Removing n-tuple points, Trans. Amer. Math. Soc., 298 (1986), 83-102.

[Ecc79] P. J. Eccles, Multiple points of codimension one immersions, in Proceedings of 1979 Siegen Topology Symposium, Lecture Notes in Math., vol. 788, pages 23-28, Springer-Verlag, 1979. 
[Ecc80] _ Multiple points of codimension one immersions of oriented manifolds, Math. Proc. Camb. Phil. Soc., 87 (1980), 213-220.

[Fra71] G. K. Francis, Generic homotopies of immersions, Indiana Univ. Math. J., 21 (1972), 1101-1112.

[Fre78] M. Freedman, Quadruple points of 3-manifolds in $S^{4}$, Comm. Math. Helv., 53 (1978), 385-394.

[HH85] J. Hass and J. Hughes, Immersions of surfaces in 3-manifolds, Topology, 23 (1985), 92-112.

[HM85] J. Hughes and P. Melvin, The Smale invariant of a knot, Comm. Math. Helv., 60 (1985), 615-627.

[KM58] M. Kervaire and J. Milnor, Homotopy groups, Bernoulli numbers, and a theorem of Rochlin, in Proceedings of the International Congress of Mathematicians, 1958.

[MB81] N. Max and T. Banchoff, Every sphere eversion has a quadruple point, in (R. Sachsteder, D. N. Clark, G. Pecelli, editors), Contributions to Analysis and Geometry, pages 191-209, Johns Hopkins University Press, 1981.

[Sma59] S. Smale, The classification of immersions of spheres in Euclidean space, Annals of Math., 69 (1959), 327-344.

[Ste51] N. Steenrod, Fiber Bundles, Princeton University Press, 1951.

[Wel66] R. Wells, Cobordism groups of immersions, Topology, 5 (1966), 281-294.

Received December 5, 1989 and in revised form May 6, 1991.

BROWN UNIVERSITY

PROVIDENCE, RI 02912 


\title{
PACIFIC JOURNAL OF MATHEMATICS
}

Founded by

\author{
E. F. BECKENBACH (1906-1982) F. WolF (1904-1989) \\ EDITORS
}

\author{
V. S. VARADARAJAN \\ (Managing Editor) \\ University of California \\ Los Angeles, CA 90024-1555 \\ vsv@math.ucla.edu \\ Herbert Clemens \\ University of Utah \\ Salt Lake City, UT 84112 \\ clemens@math.utah.edu \\ F. Michael Christ \\ University of California \\ Los Angeles, CA 90024-1555 \\ christ@math.ucla.edu \\ ThOMAs ENRIGHT \\ University of California, San Diego \\ La Jolla, CA 92093 \\ tenright@ucsd.edu
}

\author{
Nicholas ERCOLANI \\ University of Arizona \\ Tucson, AZ 85721 \\ ercolani@math.arizona.edu \\ R. FINN \\ Stanford University \\ Stanford, CA 94305 \\ finn@gauss.stanford.edu \\ VAUGHAN F. R. JONES \\ University of California \\ Berkeley, CA 94720 \\ vfr@math.berkeley.edu \\ Steven KerckhofF \\ Stanford University \\ Stanford, CA 94305 \\ spk@gauss.stanford.edu
}

C. C. Moore

University of California

Berkeley, CA 94720

MaRTin SCHARLEMANN

University of California

Santa Barbara, CA 93106

mgscharl@henri.ucsb.edu

HAROLD STARK

University of California, San Diego

La Jolla, CA 92093

\author{
UNIVERSITY OF ARIZONA \\ UNIVERSITY OF BRITISH COLUMBIA \\ CALIFORNIA INSTITUTE OF TECHNOLOGY \\ UNIVERSITY OF CALIFORNIA \\ MONTANA STATE UNIVERSITY \\ UNIVERSITY OF NEVADA, RENO \\ NEW MEXICO STATE UNIVERSITY \\ OREGON STATE UNIVERSITY
}

\section{SUPPORTING INSTITUTIONS}

UNIVERSITY OF OREGON

UNIVERSITY OF SOUTHERN CALIFORNIA

STANFORD UNIVERSITY

UNIVERSITY OF HAWAII

UNIVERSITY OF TOKYO

UNIVERSITY OF UTAH

WASHINGTON STATE UNIVERSITY

UNIVERSITY OF WASHINGTON 


\section{PACIFIC JOURNAL OF MATHEMATICS}

Volume $156 \quad$ No. $1 \quad$ November 1992

Enveloping algebras of Lie groups with discrete series

Nguyen HuU AnH and VuOng Manh Son

Asymptotic behavior of eigenvalues for a class of pseudodifferential operators on $\mathbf{R}^{n}$

JUNICHI ARAMAKI

A hybrid of theorems of Vinogradov and Piatetski-Shapiro

ANTAL BALOG and JOHN BENJAMIN FRIEDLANDER

Chaos in terms of the map $x \rightarrow \omega(x, f)$

ANDREW MiCHAEL BRUCKNER and JACK GARY CEDER

Local real analytic boundary regularity of an integral solution operator 97 of the $\bar{\partial}$-equation on convex domains

ZHENHUA CHEN

On some properties of exhaustion maps between bounded domains

Chi Keung Cheung

A generalization of maximal functions on compact semisimple Lie groups

HENDRA GUNAWAN

Stability of nonsingular group orbits

CLARK DEAN HORTON

Bordism and regular homotopy of low-dimensional immersions

JOHN FORBES HuGHES

On six-connected finite $H$-spaces

JAMES PEICHENG LiN and Frank WiLliams 